\title{
OPTIMIZING ASSEMBLY LINE SUPPLY BY INTEGRATING WAREHOUSE PICKING AND FORKLIFT ROUTING USING SIMULATION
}

\author{
Stefan Vonolfen \\ Monika Kofler \\ Andreas Beham \\ Michael Affenzeller
}

Heuristic and Evolutionary Algorithms Laboratory

University of Applied Sciences Upper Austria

School of Informatics/Communications/Media

Softwarepark 11, A-4232 Hagenberg, AUSTRIA

\author{
Werner Achleitner \\ Rosenbauer International AG \\ Paschingerstrasse 90 \\ A-4060 Leonding, AUSTRIA
}

\section{EXTENDED ABSTRACT}

The significance of system orientation in production and logistics optimization has often been neglected in the past. An isolated view on single activities may result in globally suboptimal performance. We consider a manufacturing process where assembly lines are supplied from a central logistics center.

Material handling is an important activity in manufacturing. Raw materials and work-in-process materials are stored and picked in warehouses and transported within production plants or distribution centers. In manufacturing, material handling can make up to $20 \%-50 \%$ of the total operating expenses as pointed out by Tompkins et al. (2010). In this work we deal with two aspects of material handling in a production plant: warehouse picking and forklift routing.

Our research was motivated by an industrial application where multiple production lines are supplied from various warehouses at the production plant at Rosenbauer. The material is stored in high rack storage areas and picked by employees utilizing forklifts. After the parts have been picked in the warehouse, they are handed over to four smaller forklifts at predefined intermediate storage places. These then transport the picked parts to workstations in the production line. The transport demands of parts from the warehouses to the workstations and between workstations are highly dynamic and transport orders and routes are not yet optimized. Because in our scenario the arrival of the transportation requests depends on the warehouse picking process, it makes sense to consider the problem as an integrated formulation.

In the investigated scenario, the primary goal is to guarantee an optimal flow of material within Rosenbauer's largest production plant. Most importantly, the individual workstations and assembly lines need to be supplied with parts from the warehouses and semi-finished goods need to be transported between production areas and back into storage. Production plans and their associated bills of materials are available with daily precision in the enterprise resource planning system (ERP). However, these daily production plans are highly dynamic and rush orders or plan changes occur frequently during the day, thus triggering new transport request from storage into production.

Previous work has shown potential for improving pick efficiency at the plant in Leonding via storage assignment optimization (Kofler et al. 2011). In many order picking environments the travel time to retrieve an order has been found to be the largest component of labor, amounting to $50 \%$ or more of total order picking time (Tompkins et al. 2010). However, previous studies with Rosenbauer have shown, that not considering the interdependencies with the in-house transport increases uncertainty about how the entire process will be affected by storage optimization. Thus, the integrated view of picking and routing is crucial for the practical realization of the optimized process. 
The different steps, such as storage, picking and transport of work-in-process materials to and from the assembly lines, strongly influence each other. For instance, if the picking process batches orders that need to be transported to the same target, a reduction of travel distances can be achieved. The individual problems are coupled and validated via simulation, which leads to more robust and applicable results in practice.

In this work we perform a simulation study to show how the integrated optimization of warehouse picking and forklift routing leads to a more efficient material handling in our industrial scenario. We show, that the efficiency of the forklift routes transporting the goods from the intermediate storage to the workstations is strongly dependent on the upstream picking processes in the warehouse and analyze the dependencies. The warehouse picking and forklift routing optimization models are coupled by means of simulation and are optimized using metaheuristic algorithms which are implemented in HeuristicLab (Wagner 2009) in a simulation optimization approach.

Integrated formulations of warehousing and internal transport have already been considered for container terminals which are characterized by a tight coupling of the individual processes and also a high degree of automation. Bish (2003) consider loading and unloading of containers from and to ships and storing the containers in the terminal yard. The ships are served by multiple quay cranes and the containers are moved by a fleet of vehicles. Lee et al. (2009) present an integrated model of yard truck scheduling and the storage allocation in a container terminal and develop a hybrid insertion heuristic. Cao et al. (2010) formulate a mixed-integer programming model for this problem and solve it using Benders' decomposition. We apply the idea of an integrated view of warehousing and transport to the application domain of production logistics.

One of the main research contributions of this paper is the integrated simulation and optimization of warehouse picking and forklift routing to streamline the assembly line supply. We illustrate our approach on a complex production environment dealing with the construction of firefighting vehicles at Rosenbauer. Our results indicate that warehouse optimization can lead to a more efficient transport in an integrated problem formulation.

\section{ACKNOWLEDGMENTS}

Part of the work described in this article was done within the Regio 13 program sponsored by the European Regional Development Fund and by Upper Austrian public funds. Part of the work was carried out within the Josef Ressel Centre for Heuristic Optimization and supported by the Austrian Research Promotion Agency (FFG).

\section{REFERENCES}

Bish, E. 2003. "A multiple-crane-constrained scheduling problem in a container terminal". European Journal of Operational Research 144 (1): 83-107.

Cao, J., D. Lee, J. Chen, and Q. Shi. 2010. "The integrated yard truck and yard crane scheduling problem: Benders decomposition-based methods". Transportation Research Part E: Logistics and Transportation Review 46 (3): 344-353.

Kofler, M., A. Beham, S. Wagner, M. Affenzeller, and W. Achleitner. 2011, aug.. "Re-warehousing vs. healing: Strategies for warehouse storage location assignment". In Logistics and Industrial Informatics (LINDI), 2011 3rd IEEE International Symposium on, 77 -82.

Lee, D., J. Cao, Q. Shi, and J. Chen. 2009. "A heuristic algorithm for yard truck scheduling and storage allocation problems". Transportation Research Part E: Logistics and Transportation Review 45 (5): 810-820.

Tompkins, J., J. White, Y. Bozer, and J. Tanchoco. 2010. Facilities planning. 4th ed. New York: Wiley.

Wagner, S. 2009. Heuristic Optimization Software Systems - Modeling of Heuristic Optimization Algorithms in the HeuristicLab Software Environment. Ph. D. thesis, Johannes Kepler University, Linz, Austria. 